\title{
Clinico-radiological-pathological correlation in eumycetoma spectrum: Case series
}

\author{
Taruna Yadav, ${ }^{1}$ (D) Virendra Kumar Meena, ${ }^{1}$ (D) Minhaj Shaikh, ${ }^{1}$ (i) Sudeep Khera, ${ }^{2}$ (i) Binit Sureka, ${ }^{1}$ \\ Dawan Garg, ${ }^{1}$ (D) Pushpinder Singh Khera ${ }^{1}$ \\ ${ }^{1}$ Department of Diagnostic and Interventional Radiology, All India Institutes of Medical Sciences, Jodhpur, Rajasthan, India \\ ${ }^{2}$ Department of Pathology, All India Institutes of Medical Sciences, Jodhpur, Rajasthan, India
}

\begin{abstract}
Maduramycosis or mycetoma is one of the chronic granulomatous diseases commonly seen in tropical regions. Microbiological cultures and biopsy are carried out for the definitive diagnosis of the disease, but they are time-consuming methods. The present study aims to correlate clinical, radiological and pathological features in eumycetoma cases to emphasize the role of "dot in circle" sign leading to early imaging-based diagnosis. Imaging plays an important role in early diagnosis of mycetoma, which has therapeutic implications. "Dot in circle" sign is a recently described sign in mycetoma cases on ultrasound (USG) and magnetic resonance imaging (MRI). We diagnosed four cases of eumycetoma based on clinical and imaging features, which were confirmed with histopathology. The differential diagnosis, which may mimic this sign is also discussed. The "dot in circle" sign is seen on both ultrasound and MRI. This sign is highly specific for mycetomas. Knowledge of this sign can help in early diagnosis of mycetomas preventing misdiagnosis and further complications.
\end{abstract}

Keywords: Dot in circle; granulomatous; magnetic resonance imaging; mycetoma; ultrasound.

Cite this article as: Yadav T, Meena VK, Shaikh M, Khera S, Sureka B, Garg P, et al. Clinico-radiological-pathological correlation in eumycetoma spectrum: Case series. North Clin Istanb 2020;7(4):400-406.

$\mathrm{I}^{\mathrm{n}}$ n 1842, Gill first recognized Madura foot or mycetoma as a disease entity in Madurai, India, and in 1880, Carter coined the term "Mycetoma" [1]. Mycetoma may arise from the Eumyces, or true fungi (eumycetoma), or Actinomyces, filamentous bacteria of the order Actinomycetales (actinomycetoma) [2]. Mycetoma occurs more commonly in tropical climate regions. It frequently involves exposed areas of the body which come in direct contact with soil, so commonly occurs in feet, lower legs and hands. Thorn prick with direct inoculation of the infecting organism is the most common mechanism. Mycetoma is a chronic localized granulomatous disease, which is slowly progressive in nature. It is characterized by excessive formation of granulation tissue and discharging sinuses; however, in the later course of the disease, it may also involve the bone. The appearance of discharging sinuses is very important for making a clinical diagnosis of mycetoma, but in the early stages of the disease without the formation of discharging sinuses, clinically, it looks similar to chronic bacterial infections or soft-tissue neoplasms. Thus, imaging plays an essential role in early diagnosis [2]. USG and MRI both demonstrate a recently described "dot in circle" sign (Fig. $1)$. The specificity of the "dot in circle" sign on MRI and USG for the detection of mycetoma is high [3-5].

\section{CASE REPORTS}

Case 1 - A 35-year-old male, a teacher by occupation, presented with complaints of swelling in dorsum of right foot over the region of middle toe (Fig. 2A), which was

Received: January 23, 2019 Accepted: May 14, 2019 Online: July 12, 2019

Correspondence: Taruna YADAV, MD. Department of Diagnostic and Interventional Radiology, All India Institutes of Medical Sciences, Jodhpur, Rajasthan, India

Tel: 9540950997 e-mail: drtanu.taruna@gmail.com

(c) Copyright 2020 by Istanbul Provincial Directorate of Health - Available online at www.northclinist.com 


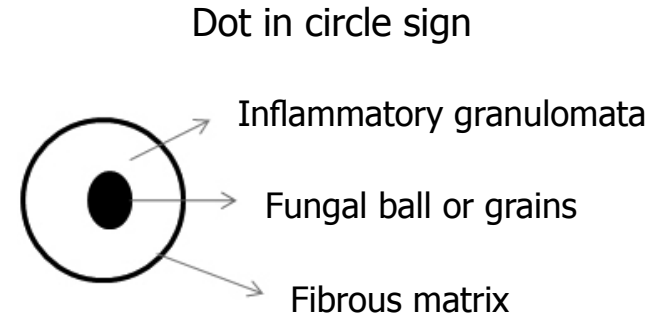

FIGURE 1. Components forming dot in circle on imaging.

gradually increasing in size over a period of two years. He had a history of thorn prick over middle toe while gardening in his farmhouse. The swelling was also associated with discharge from multiple sinuses. The radiograph of the foot did not reveal any abnormality except soft-tissue density over the dorsum of the middle toe (Fig. 2B). During further workup, the ultrasound showed multiple lesions with hyperechoic foci in the center and hypoechoic wall in the periphery of lesions (Fig. 2C). MRI of foot confirmed multiple hyperintense lesions with central hypointense dots and surrounding hypointense tissue (Fig. 2D, E). Bone marrow involvement or osteomyelitis was absent.
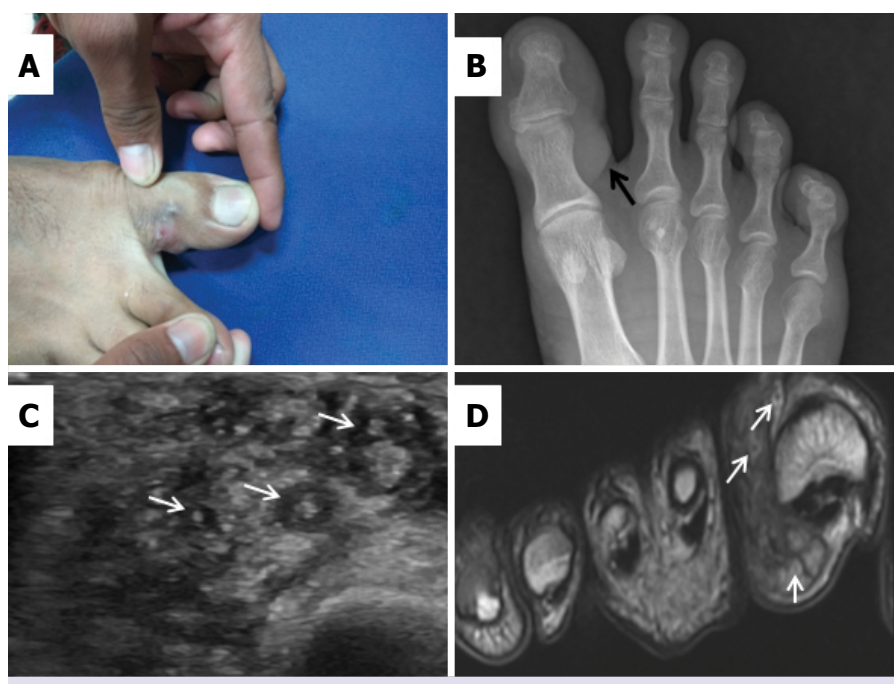

FIGURE 3. Eumycetoma of the great toe. (A) Swelling at the lateral aspect of the great toe with discharging sinus and foci of hyperpigmentation. (B) Radiograph of the foot (AP view) image shows soft-tissue density over the lateral aspect of the great toe (black arrow). (C) On ultrasound, multiple lesions (white arrows) are evident with thick hypoechoic walls and hyperechoic foci in the center. (D) Axial T2-weighted MRI shows multiple hyperintense lesions with central hypointense dots (white arrows).

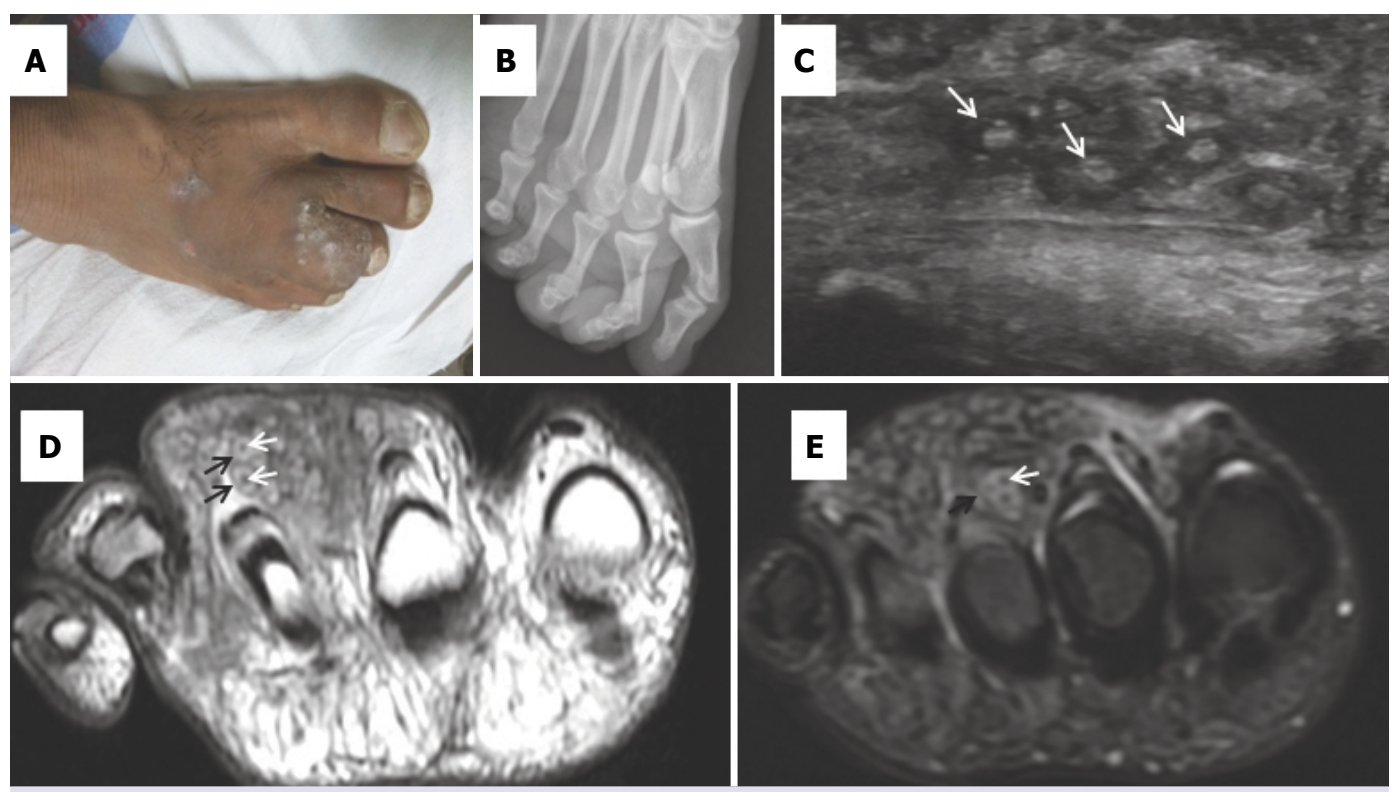

FIGURE2. Eumycetoma of the foot. (A) Soft tissue swelling at the dorsum of right middle toe and foot region with multiple encrusted skin sinuses. (B) Radiograph of the foot (AP view) appears normal except a soft-tissue density shadow over heads of $2-4^{\text {th }}$ metatarsals. (C) Ultrasound image shows multiple lesions (white arrows) with hypoechoic walls (circle) and hyperechoic foci in the center (dots) giving the appearance of "dot in circle". (D, E) Axial T2-weighted (D) and STIR (E) MRI images showing multiple hyperintense lesions with hypointense periphery (black arrows) with central hypointense dots (white arrow) giving the appearance of "dot in circle". 

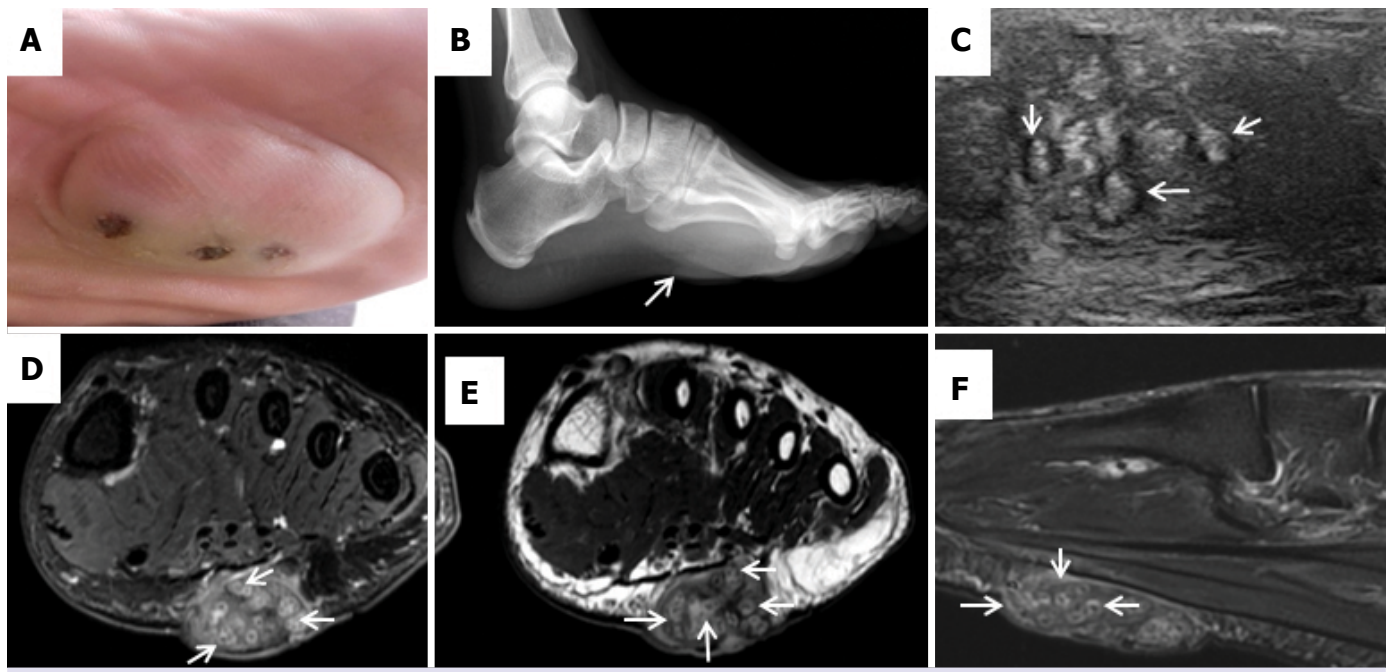

FIGURE 4. Eumycetoma at the plantar aspect of the foot. (A) A protuberant swelling at the plantar aspect of foot with few encrusted sinuses. (B) Lateral radiograph of the foot appears normal except a soft-tissue density shadow on the plantar aspect (arrow). (C) On high-resolution ultrasound, multiple clustered lesions are noted, which have a hypoechoic periphery and hyperechoic center. (D-F) Axial STIR (D), T2WI (E), and Sagittal STIR (F) MRI images show multiple hyperintense lesions with central hypointense dots (white arrows) showing typical "dot in circle" sign in the subcutaneous plane.
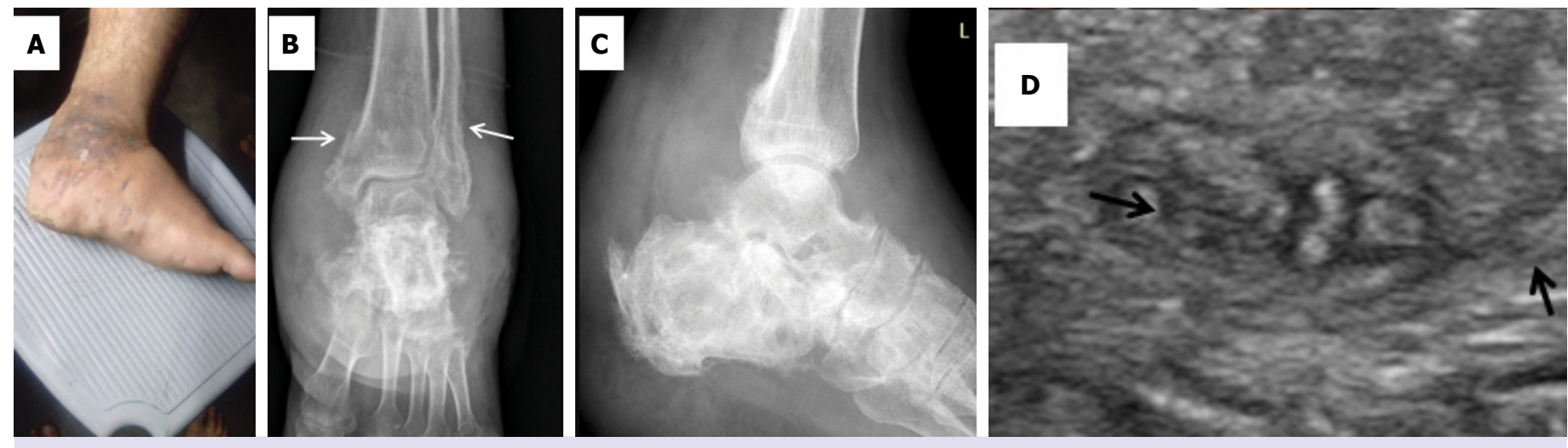

FIGURE 5. Eumycetoma with bone involvement. (A) Soft-tissue swelling at the ankle on the medial side, extending into plantar aspect of foot with few encrusted sinuses and hyperpigemted patches in the skin. (B, C) AP and lateral radiographs of ankle show multiple osteolytic and sclerotic lesions in calcaneum with periosteal reaction (white arrows) at the lower end of tibia and fibula. (D) Ultrasound image shows multiple lesions with dot in circle sign (black arrows).

Case 2 - A 27-year-old male came with complaints of soft-tissue swelling and few discharging sinuses in the dorsolateral aspect of right great toe (Fig. 3A). He noticed this swelling six months back. He had no significant occupational history or history of thorn prick. Foot radiograph showed mild soft-tissue density along the proximal phalanx in the lateral aspect of the great toe (Fig. 3B). On ultrasound, multiple hypoechoic lesions with hyperechoic foci in center were seen (Fig. 3C). On MRI of the foot, multiple hyperintense lesions with central hypointense dots consistent with the dot in circle sign were seen (Fig. 3D).

Case 3 - A 32-year-old male presented with a soft-tissue swelling in the plantar aspect of left foot (Fig. 4A). The swelling was partially exophytic in appearance. Three discharging sinuses were present at the dome of swelling. He was a farmer by occupation. Foot radiograph showed no significant abnormality except soft-tissue density shadow (Fig. 4B). On ultrasound, multiple clustered lesions with the hypoechoic wall in 

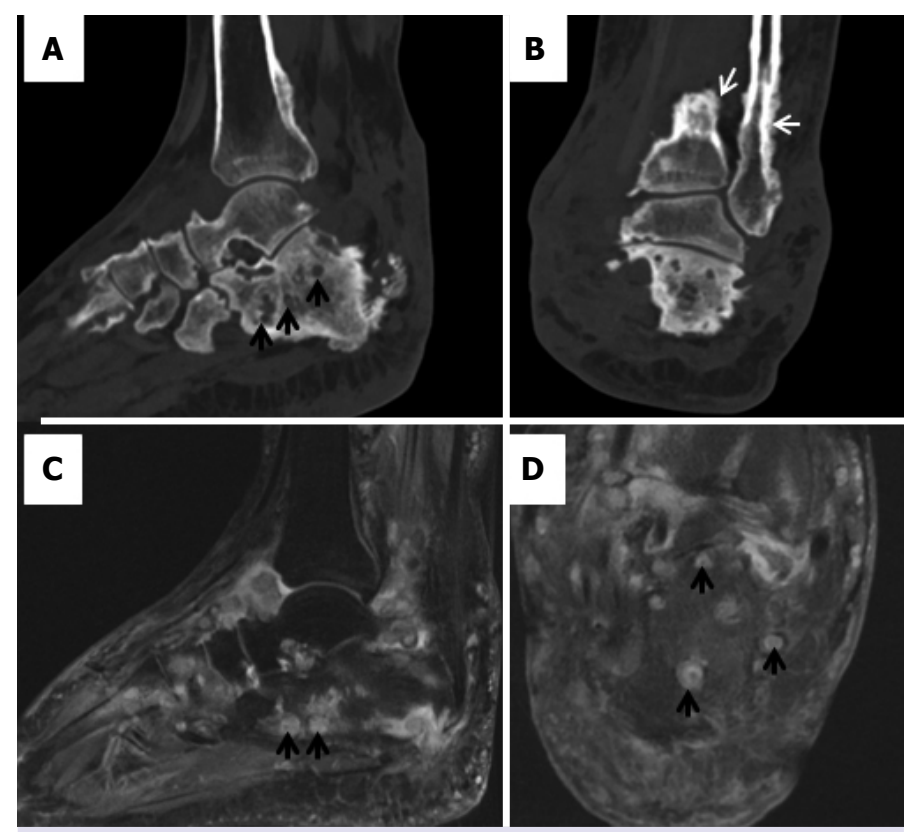

FIGURE 6. CT and MRI of the ankle of the patient with eumycetoma in Figure 5. (A, B) Sagittal and coronal reformats of CT of ankle show multiple osteolytic areas (black arrows) in calcaneum with periosteal reaction at the lower end of tibia and fibula (white arrows) with soft-tissue involvement, respectively. (C, D) Saggital and coronal STIR MRI images show multiple lesions in calcaneum and soft tissues with a dot in circle sign (arrows).

the periphery and hyperechoic foci in the center were seen (Fig. 4C). MRI of foot demonstrated multiple lesions with the dot in circle sign (Fig. 4D-F).

Case 4 - A 37-year-old male had a history of minor trauma over the left ankle 10 years back, which was fol- lowed by swelling over the left ankle and was associated with black grain discharge (Fig. 5A). Foot radiographs revealed multiple osteolytic lesions in calcaneum, associated with the periosteal reaction in tibia and fibula (Fig. 5B, C). Ultrasound of ankle showed few ill-defined lesions with hyperechoic foci in the center and hypo echoic wall in periphery (Fig.5D). CT ankle confirmed multiple osteolytic lesions in calcaneum with periosteal reaction in tibia and fibula (Fig. 6A, B) confirmed. MRI ankle demonstrated multiple hyperintense lesions with a dot in circle sign in both soft tissues and in calcaneum (Fig. 6C, D).

In all four cases, the final diagnosis was eumycetoma, which was confirmed on histopathology (Fig. 7). In the first three cases, surgery was carried out, followed by antifungal therapy. In the $4^{\text {th }}$ case, the patient did not give consent for surgery and is on antifungal therapy for the last eight months.

Consent was obtained from each patient for this report.

\section{DISCUSSION}

Mycetoma is a chronic granulomatous infection of the dermis and epidermis, which may arise from the bacteria Actinomyces (Actinomycetoma) or by true fungi (eumycetoma) [6-9]. Mycetoma is more prevalent in tropical regions and is endemic in India. All of our cases belonged to western Rajasthan, in India, which is a tropical area. Eumycetoma is more prevalent in areas with less rainfall, while actinomycetoma is more prevalent in areas with heavy rainfall. It is presumed that there is a direct introduction of infecting organisms after trauma

\section{A}

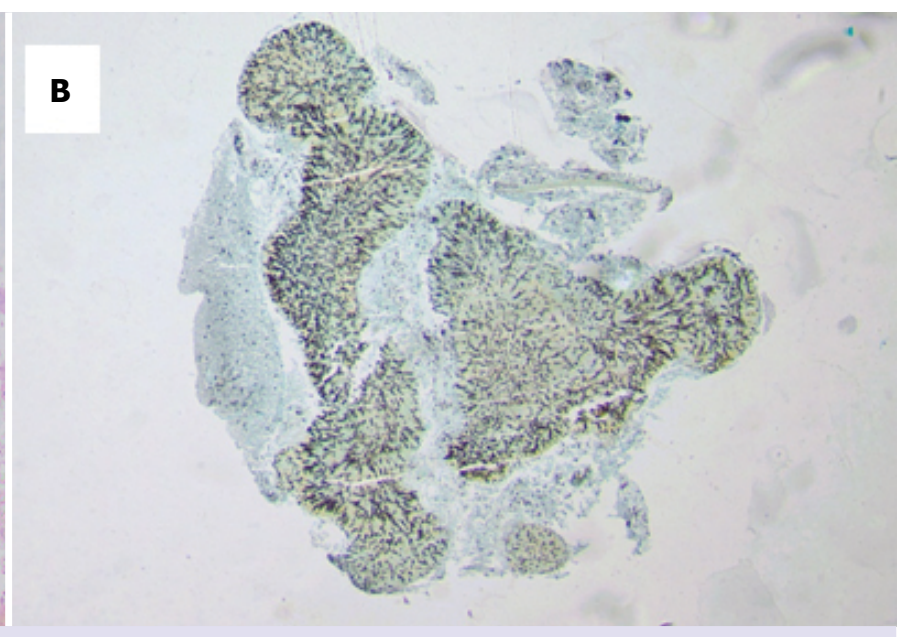

FIGURE 7. Eumycetoma on histology. (A) Haematoxylin and eosin (H\&E) stain, low magnification, shows a cluster of fungal hyphae surrounded by the inflammatory response (B) GMS staining positive for fungal hyphae. 
or direct penetration of the skin with sharp objects. This infection more commonly involves feet because they are more prone to trauma. First of all, soft-tissue swelling occurs with inflammation in surrounding tissues due to the formation of granulation tissue. This granulation tissue further leads to the formation of multiple discharging sinuses. Bone involvement may occur in the later course of the disease after many years $[1,6]$.

Clinically, when any patient presents with soft-tissue swelling and discharging sinuses, then, diagnosis of mycetoma should be considered [3]. Mycetoma is a slowly progressive disease. If proper treatment is not initiated in the early stages of the disease then, it may lead to multiple complications, such as osteomyelitis, abscess formation, fistula formation and formation of multiple draining sinuses tracts with deformity of the involved body part [2]. In the early stages of the disease, in the absence of discharging sinuses, a clinical diagnosis of mycetoma is difficult. It can be misdiagnosed as soft-tissue tumor or chronic indolent bacterial to tubercular infection [10]. However, imaging plays an important role in the early detection of mycetoma. "Dot in circle" sign is seen in mycetoma, and this sign is very specific for mycetoma [7] (Fig. 1). All four of our cases demonstrated "dot in circle sign" on both ultrasound and MRI. In later course of the disease, bone involvement with destruction may occur, which may require surgical management and has a bad prognosis. Thus, for a better prognosis of the patient, early detection of mycetoma is important. Imaging, particularly ultrasound and MRI, plays a crucial role in early detection of mycetoma and differentiates it from other pathologies.

Radiographs are helpful in detection of bone changes in cases of mycetoma. However, they may be normal if no bone involvement is present, especially in the early stage of the disease. Common findings present on radiographs are bone sclerosis, periosteal reaction, bone cavities, cortical scalloping, osteoporosis and soft-tissue thickening [7]. Sometimes, bone changes on radiographs are helpful in the differentiation of the eumycetoma and actinomycetoma. Lesions of eumycetoma have a tendency to form few cavities in bone, which are more than one centimeter in diameter. Bone lesions in actinomycetes usually have more number of cavities but are small in size, which leads to moth-eaten appearance [2]. Bagi et al. [7] have given a radiographic classification based on the extent of the bone involvement (stages 0-6). Computerized tomography (CT) scan depicts the extent of bone changes involvement better than radiographs. Initially, Czechowski et al. [8], in 2001, observed small foci of low-signal intensity on both T1- and T2- weighted images and assumed that the low-signal intensity of these foci is due to susceptibility from the metabolic products of the "grains".

In 2003, Sarris et al. [4] described the "dot in circle" sign, as tiny hypointense foci within the hyperintense spherical or circular lesions on T2W and STIR sequences. Further correlation with histopathological findings revealed that high signal intensity on MRI is due to inflammatory granulomata, central low signal intensity part is composed of fungal balls or grain, and

TABLE 1. Differences between eumycetoma and actinomycetoma

\begin{tabular}{|c|c|c|}
\hline Features & Eumycetoma & Actinomycetoma \\
\hline Etiology & True fungi & Filamentous bacteria \\
\hline Epidemiology & In drier areas & With abundant rainfall \\
\hline Course of disease & Slowly progressive & Rapidly progressive, with more destruction \\
\hline Size and Color of grains & Coarse, Black or pale & Fine, white, yellow or red \\
\hline Radiographic changes & $\begin{array}{l}\text { Few cavities in the bone that are } \\
\geq 1 \mathrm{~cm} \text { in diameter }\end{array}$ & $\begin{array}{l}\text { Early bone involvement. Smaller but more numerous } \\
\text { cavities, leading to a moth-eaten appearance }\end{array}$ \\
\hline Muscle involvement & Uncommon & May be present \\
\hline Ultrasound & $\begin{array}{l}\text { The grains are many and they appear as } \\
\text { very sharp hyperechogenicity. Single or } \\
\text { multiple, hypoechoic, thick-walled } \\
\text { cavities with no acoustic enhancement }\end{array}$ & $\begin{array}{l}\text { The grains are fine, hazy, closely aggregated and } \\
\text { commonly settle at the bottom of the cavities }\end{array}$ \\
\hline
\end{tabular}



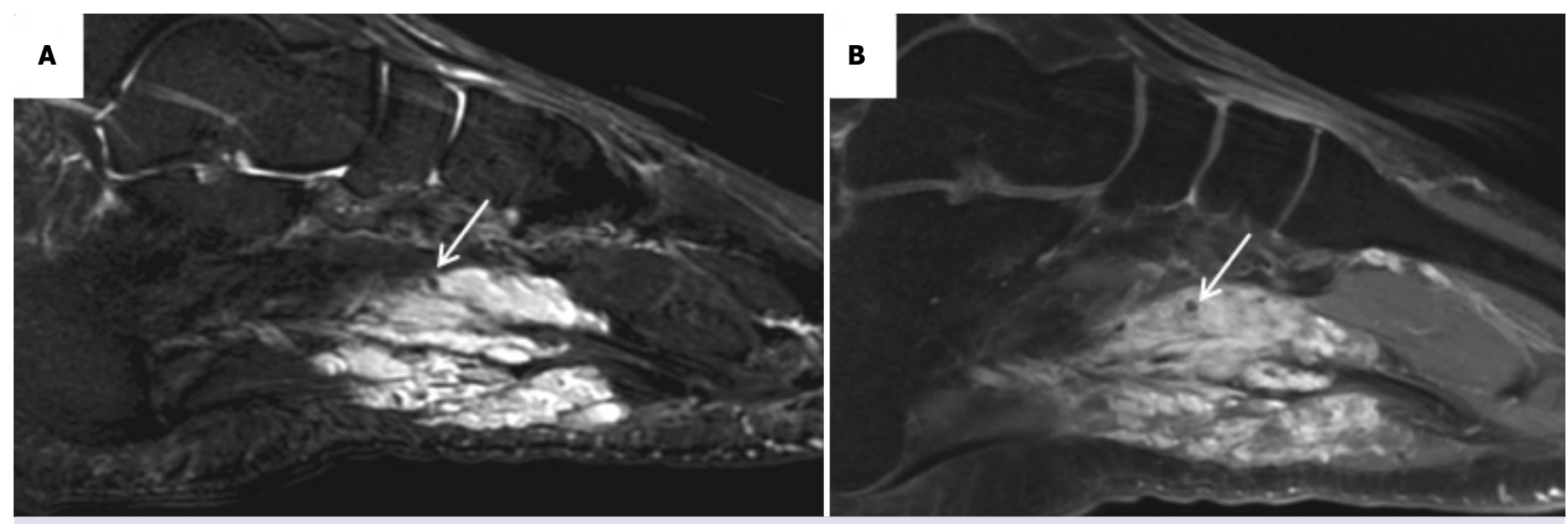

FIGURE 8. Dot in circle sign mimic - Phleboliths in a case of soft-tissue hemangioma in the foot (case not described in this series). (A) Sagittal STIR and (B) post-contrast T1W images show small phlebolith (arrow) in a hemangioma on the plantar aspect of the foot, which can give a similar appearance as a dot in circle sign.

outer hypointensity is due to the fibrous matrix. Finally, they, too, concluded that the "dot in circle" sign is a highly specific sign for mycetoma. Later, the "dot in circle" sign was also reported by Kumar et al. [3], in 2007 and Lee Parker et al. [9], in 2009. T1-weighted post-contrast (gadolinium) images may also show dot in circle sign; however, do not appear to have an advantage over sequences without contrast. We did not do post-contrast MRI in any of our cases. Dot in circle sign can be seen in mycetomas involving soft tissues, as well as bones, as seen in our case number four.

Ultrasound features of mycetoma were first described by Fahal et al,, and they described that hyper-reflective echoes represent grains which are surrounded by hypoechoic tissue $[4,11]$. Eumycetoma grains generally produce sharp hyperechoic foci, while hyperechoic foci in actinomycetoma are usually seen at the bottom of the lesions. "Dot-in-circle" sign in USG is similar to the MRI. Differences between eumycetoma and actinomycetoma are listed in Table 1.

Histopathological examination of lesion reveals that lesion is composed of grains of fungal hyphae or bacteria in the background of granulomatous reaction $[12,13]$. Multiple methods are available for distinguishing actinomycetoma from eumycetoma like gram stain, periodic acid-Schiff, Gomori methenamine silver and lactophenol blue stain [13]. Biopsy or staining and culture usually give the definitive diagnosis, but these are time-consuming methods. Sometimes, making the correct diagnosis with the help of biopsy and staining is difficult, mainly in fastidious organisms.

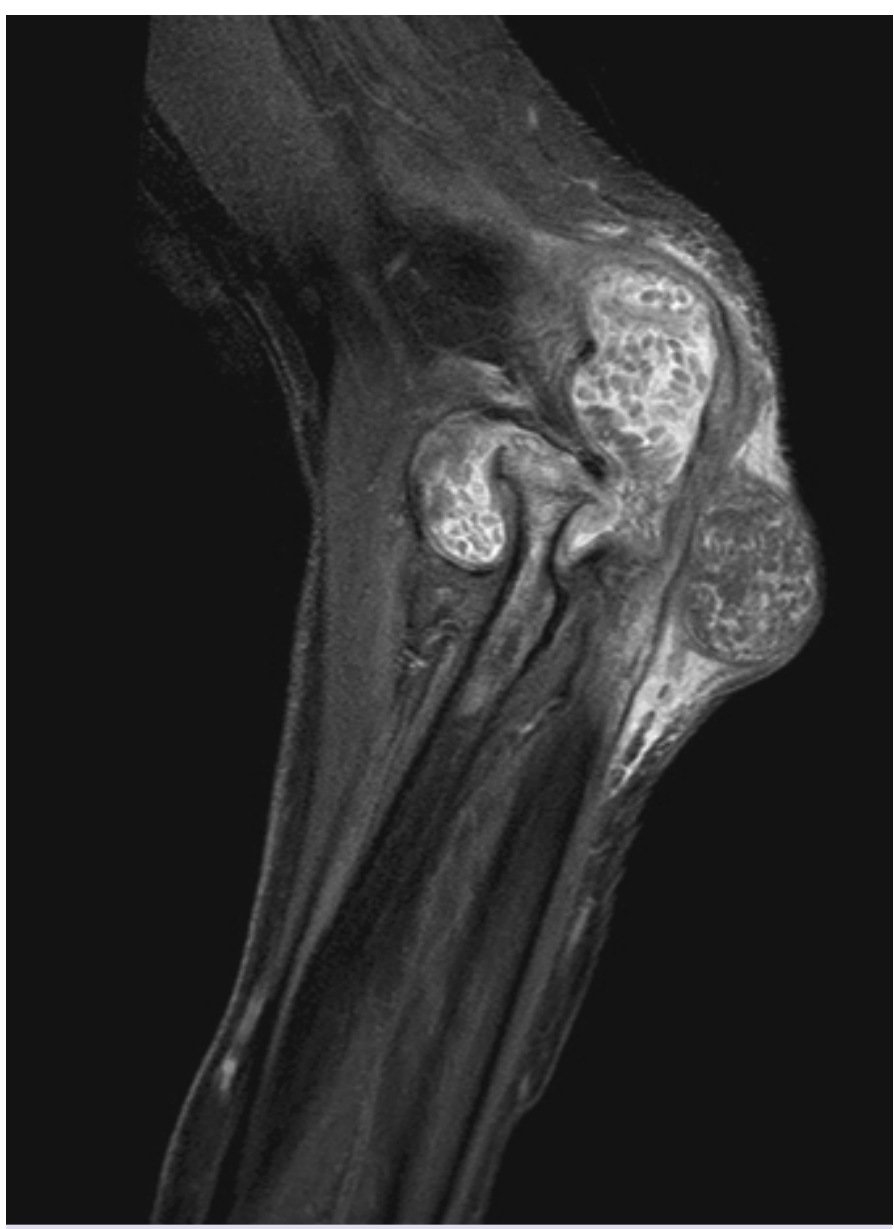

FIGURE 9. Dot in circle sign mimics - Rice bodies (case not described in this series). Oblique sagittal STIR image of the elbow joint in a patient with articular tuberculosis. Multiple small hypointense nodular "rice bodies" are seen distending the joint and can mimic as a dot in circle sign. 
Imaging mimics for dot in circle sign are phleboliths in hemangiomas and rice bodies. Sometimes, hemangiomas or low flow vascular malformations may mimic as a dot in circle sign (Fig. 8) due to the presence of phleboliths, which are seen as hypointense foci in the background of the hyperintense lesion. However, clinical history, fewer number of the dot in circles, absence of sinuses, and absence of inflammation in surrounding tissues should favour the diagnosis of vascular malformation.

Rice bodies are also hypointense nodular foci formed due to chronic synovial inflammation. These are seen in the synovial fluid of patients of rheumatoid arthritis or tuberculosis $[5,10]$ (Fig. 9).

\section{Conclusion}

To conclude, the "dot in circle" sign is seen on both ultrasound and MRI. This sign is highly specific for mycetomas. Knowledge of this sign can help in early diagnosis of mycetomas preventing misdiagnosis and further complications.

Informed Consent: Written informed consent was obtained from the patients for the publication of the case report and the accompanying images.

Conflict of Interest: No conflict of interest was declared by the authors.

Financial Disclosure: The authors declared that this study has received no financial support.

Authorship Contributions: Concept - TY, PSK, BS; Design - TY, PSK, PG; Supervision - TY, BS, PG; Data collection and/or processing - TY, VKM, MS, SK; Analysis and/or interpretation - TY, VKM, MS, SK; Literature review - MS, VKM, SK; Writing - TY; Critical review - PSK, PG, BS.

\section{REFERENCES}

1. Carroll DS. Mycetoma pedis. Radiology 1949;53:81-4. [CrossRef]

2. Lewall DB, Ofole S, Bendl B. Mycetoma. Skeletal Radiol 1985;14:25762. [CrossRef]

3. Kumar J, Kumar A, Sethy P, Gupta S. The dot-in-circle sign of mycetoma on MRI. Diagn Interv Radiol 2007;13:193-5.

4. Sarris I, Berendt AR, Athanasous N, Ostlere SJ; OSIRIS collaborative study group. MRI of mycetoma of the foot: two cases demonstrating the dot-in-circle sign. Skeletal Radiol 2003;32:179-83. [CrossRef]

5. Cherian RS, Betty M, Manipadam MT, Cherian VM, Poonnoose PM, Oommen AT, et al. The "dot-in-circle" sign - a characteristic MRI finding in mycetoma foot: a report of three cases. Br J Radiol 2009;82:6625. [CrossRef]

6. Negroni R, López Daneri G, Arechavala A, Bianchi MH, Robles AM. Clinical and microbiological study of mycetomas at the Muñiz hospital of Buenos Aires between 1989 and 2004. [Article in Spanish]. Rev Argent Microbiol 2006;38:13-8.

7. Abd El Bagi ME. New radiographic classification of bone involvement in pedal mycetoma. AJR Am J Roentgenol 2003;180:665-8. [CrossRef]

8. Czechowski J, Nork M, Haas D, Lestringant G, Ekelund L. MR and other imaging methods in the investigation of mycetomas. Acta Radiol 2001;42:24-6. [CrossRef]

9. Parker L, Singh D, Biz C. The dot-in-circle sign in Madura foot. J Foot Ankle Surg 2009;48:690.e1-690.e6905. [CrossRef]

10. Hsu CY, Lu HC, Shih TT. Tuberculous infection of the wrist: MRI features. AJR Am J Roentgenol 2004;183:623-8. [CrossRef]

11. Fahal AH, Sheik HE, Homeida MM, Arabi YE, Mahgoub ES. Ultrasonographic imaging of mycetoma. Br J Surg 1997;84:1120-2. [CrossRef]

12. Magana M. Mycetoma, some clinical and histopathological features. Turk J Dermatopathol 1994;3:94.

13. Pilsczek FH, Augenbraun M. Mycetoma fungal infection: multiple organisms as colonizers or pathogens?. Rev Soc Bras Med Trop 2007;40:463-5. [CrossRef] 